\title{
Two sides of weight bias in adolescent binge-eating disorder: adolescents' perceptions and maternal attitudes
}

Anne Pötzsch, M.Sc. ${ }^{1 *}$, Almut Rudolph, Ph.D. ${ }^{2}$, Ricarda Schmidt, Ph.D. ${ }^{1}$, and Anja Hilbert, Ph.D. ${ }^{1}$

Running title: Weight bias in families with BED

${ }^{1}$ Leipzig University Medical Center, Integrated Research and Treatment Center AdiposityDiseases, Departments of Medical Psychology and Medical Sociology and Psychosomatic Medicine and Psychotherapy

${ }^{2}$ University of Leipzig, Department of Psychology, Clinical Psychology and Psychotherapy

* Corresponding author. Leipzig University Medical Center, Integrated Research and Treatment Center AdiposityDiseases, Departments of Medical Psychology and Medical Sociology and Psychosomatic Medicine and Psychotherapy, Philipp-Rosenthal-Strasse 27, 04103 Leipzig, Germany. Phone: +49 341 97-15363, Fax: +49 341 97-15359, E-mail: anne.poetzsch.leuna@gmail.com.

Acknowledgment:

This work was supported by the Federal Ministry of Education and Research (BMBF), Germany, FKZ: 01EO1001.

Abstract word count: 254 Number of tables: 1

Amount of words: 4043 Number of figures: 1 


\begin{abstract}
Objective: Adolescents with binge-eating disorder (BED) are suffering from weight teasing and, as found in adults with BED, are likely to internalize weight bias. Weight teasing by mothers accounts for psychopathology in overweight, but sources of stigmatization are largely unknown in BED. This study sought to address familial weight bias in adolescents with overweight and BED by examining adolescents' perceived parental weight teasing and weight bias internalization in relation to their eating disorder psychopathology and maternal stigmatizing attitudes and beliefs.
\end{abstract}

Method: Adolescents with overweight and BED (BED; $n=40)$ were compared to a sociodemographically matched group with overweight only (OW) and a normal-weight control group (NW; each $n=25$ ). They filled out the Perception of Teasing Scale, with parents as the source of teasing, the Weight Bias Internalization Scale and the Eating Disorder ExaminationQuestionnaire. Their mothers filled out the Attitudes Toward and Beliefs about Obese Persons Scales.

Results: Significantly higher perceived parental weight teasing and weight bias internalization were found in BED compared to OW and NW. Maternal stigmatizing attitudes and beliefs did not differ between groups and were not correlated with adolescents' perceptions of being stigmatized. Perceived parental weight teasing predicted adolescents' eating disorder psychopathology, however, this association was fully mediated by weight bias internalization. Discussion: These results indicate that adolescents with overweight and BED perceive weight teasing in their own families. As we found no significant association between adolescents' perceptions of being stigmatized and maternal stigmatizing attitudes, future research should examine weight-related parent-child interaction or implicit measures of stigmatizing attitudes. Keywords: weight bias, weight teasing, weight bias internalization, binge-eating disorder, adolescents, family 


\section{INTRODUCTION}

Binge-eating disorder (BED) is characterized by recurrent binge-eating episodes without regular use of inappropriate compensatory behaviors (e.g., self-induced vomiting) and has been identified in $1.6 \%$ of adolescents in the community (Swanson et al., 2011). In 2013, it was included in the fifth edition of the Diagnostic and Statistical Manual of Mental Disorders (DSM-5; APA, 2013) as a distinct diagnostic category. Additionally to higher eating disorder and general psychopathology (e.g., depression, anxiety disorders, substance abuse; Decaluwé \& Braet, 2003; Swanson et al., 2011), children and adolescents with BED showed a high comorbidity with overweight and obesity (Decaluwé et al., 2003). Based on self-report, they were found to suffer from perceptions of being stigmatized because of their higher weight status (Ashmore et al., 2008).

Perceptions of being stigmatized can involve perceived critical comments about weight and shape, weight teasing, and, at worst discrimination (for a review, see Puhl \& Latner, 2007). Cross-sectional evidence underlined strong positive associations between perceived weight teasing and eating disorder and general psychopathology including bingeeating behavior in adolescents (Libbey et al., 2008; Neumark-Sztainer et al., 2002). Based on retrospective studies in adolescents and adults (Haines et al., 2006; Hilbert, Pike, et al., 2014), perceived weight teasing was found to be a risk factor for the onset of BED. In addition to perceived weight teasing, individuals with BED tend to internalize the weight bias, and thus feel incompetent, self-hatred, and denigrated towards themselves (Durso et al., 2012). As sources of weight teasing in children and adolescents with BED are largely unknown (for a review, see Puhl \& Suh, 2015), further investigations might be relevant for explaining psychopathology.

In overweight and obesity, sources of perceived weight teasing are well-known (Puhl \& Heuer, 2009; Puhl \& Latner, 2007; Puhl \& Suh, 2015). Specifically, children and adolescents with overweight and obesity experience weight teasing from multiple sources, 


\section{WEIGHT BIAS IN FAMILIES WITH BED}

including health-care facilities, educational institutions, media, and most importantly, family members. Independent from weight status, $22 \%$ to $29 \%$ of adolescents of school- and population-based samples reported being teased by family members and received hurtful weight-related comments (Eisenberg et al., 2011). Among adults with overweight, $62 \%$ retrospectively remembered to having received negative weight- and eating-related comments by their parents during childhood multiple times ( $44 \%$ by their mothers, and $34 \%$ by their fathers; Puhl \& Brownell, 2006). Evidence showed that weight teasing by family members might be most important by playing a central role for children's emotional, cognitive, and physical development (Puhl \& Latner, 2007; Repetti et al., 2002) and was thus considered to causally affect children's and adolescents' attitudes about eating, weight, and shape (for a review, see Ventura \& Birch, 2008). However, individuals' reports of perceived weight teasing are not sufficient to objectively evaluate the phenomenon of weight teasing. Apart from adolescents' perceptions of weight teasing, knowledge about parental stigmatizing attitudes and stigmatizing beliefs may contribute to a more complete picture of weight teasing in BED, given that attitudes substantially influence future behavior (for a meta-analysis, see Kraus, 1995). Although parental stigmatizing attitudes towards weight and shape were believed to result in discrimination such as weight teasing (Puhl \& Brownell, 2003), evidence on adolescents' eating disorder psychopathology is lacking.

Both in community-based and treatment-seeking clinical samples of adults with overweight and obesity, weight bias internalization has been shown to have greater explanatory power of psychopathology over and above experiences of discrimination, stigmatizing attitudes, and body mass index (Durso \& Latner, 2008; Durso et al., 2012; Hilbert, Braehler, et al., 2014; Hübner et al., 2015; Pearl et al., 2014; Puhl et al., 2007). In contrast to discrimination and stigmatizing attitudes, where weight bias is directed to other persons, weight bias internalization is self-directed, denoting the adaptation of the weight bias to the self (Durso \& Latner, 2008). Importantly, weight bias internalization was found to act 


\section{WEIGHT BIAS IN FAMILIES WITH BED}

as a mediator between eating disorder psychopathology and weight teasing, depressive symptoms, or weight status. Cross-sectionally, weight bias internalization mediated the association between perceived weight teasing and disordered eating behavior in a student sample (O'Brien et al., 2016) as well as the relationship between depressive symptoms and disordered eating behavior in women with overweight (Sienko et al., 2016). In school-aged children, weight bias internalization mediated the relationship between weight status and restrained eating, emotional and conduct problems (Zuba \& Warschburger, 2017) suggesting that this construct may be a central indicator or precursor of psychopathology in those with overweight, while this relation warrants elaboration in individuals with BED.

While research on sources of weight bias in adolescents has either focused on adolescents' perspective or maternal perspective, this study sought to examine both perspectives of weight bias: adolescents' perceived parental weight teasing and weight bias internalization as well as maternal stigmatizing attitudes and beliefs among adolescents with overweight and BED. Although this study was initially designed to examine both maternal and paternal attitudes and beliefs, we only focused on the maternal perspective. As only $n=5$ fathers in the BED group provided data, the fathers' sample was too small for well-powered statistical analyses. To elucidate the influence of BED diagnosis and adolescents' weight status on weight bias, adolescents with overweight and BED were compared to both a matched overweight and a normal-weight control group without BED. We expected adolescents with overweight and BED to report more perceived parental weight teasing and higher weight bias internalization than adolescents with overweight and normal-weight without BED. Further, we hypothesized that adolescents who perceived more parental weight teasing and reported higher weight bias internalization would live in families with mothers with higher stigmatizing attitudes and with higher maternal stigmatizing beliefs. Finally, we expected perceived parental weight teasing to predict adolescents' eating disorder psychopathology and, moreover, weight bias internalization to be a mediator on the 


\section{WEIGHT BIAS IN FAMILIES WITH BED}

association between perceived parental weight teasing and global eating disorder psychopathology.

\section{METHODS}

\section{Participants and Recruitment}

A total of $N=90$ German adolescents aged 12 to 20 years participated in the present study. Adolescents with overweight and BED (BED; $n=40)$, adolescents with overweight and without BED (OW; $n=25)$, and adolescents with normal-weight and without any eating disorder symptoms (NW; $n=25)$ were matched by frequency according to age, sex, socioeconomic status (SES), and standard deviation score of the body mass index (BMI-SDS, only BED and OW). The BED group was recruited from a randomized-controlled trial offering cognitive-behavioral therapy for adolescents with BED (BEDA study; Hilbert, 2013). Control group participants were recruited through school and public advertisements (e.g., bulletin boards) and were offered $€ 15$ for participation.

Inclusion criteria for the BED group were a diagnosis of full-syndrome or subthreshold BED based on objective and/or subjective episodes of binge eating according to DSM-IV and DSM-5 (for greater detail, see Hilbert, 2013) and a BMI-SDS $\geq+1.28$, indicating a weight status of overweight or obesity (Wabitsch \& Kunze, 2015). Inclusion in the OW and NW control groups required a BMI-SDS $\geq+1.28(\mathrm{OW})$ and $-1.28<$ BMI-SDS < $+1.28(\mathrm{NW})$, respectively, as well as absence of current and lifetime eating disorder symptoms. Exclusion criteria for all groups were current bulimia nervosa, substance abuse, suicidal ideation, psychotic or bipolar disorder, serious unstable medical problems, intake of antipsychotic or weight-affecting drugs, psychotherapy, or behavioral weight loss treatment (e.g., diet, physical activity). 


\section{WEIGHT BIAS IN FAMILIES WITH BED}

\section{Procedure}

Ethical approval for the study was granted by the local Ethics Committee of the Medical Faculty of the University of Leipzig, Germany. All adolescents were initially screened via telephone to determine eligibility based on the inclusion and exclusion criteria. Current and lifetime eating disorder status were obtained using the diagnostic items of the German version of the well-established Eating Disorder Examination interview (EDE; Fairburn et al., 2008; German version: Hilbert \& Tuschen-Caffier, 2006). During an onsite diagnostic session (i.e., before the start of the therapy for the BED group), the full-length EDE was administered to adolescents who reported binge-eating episodes during the telephone interview, in order to ascertain a BED diagnosis. Further, written informed assent and consent were sought from adolescents $<18$ years and their parents, respectively. For participants $\geq 18$ years, written informed consent was obtained from the adolescent only. Further, adolescents' height and weight were objectively measured to calculate sex- and agespecific BMI-SDS, whereas mothers' BMI was calculated of self-reported height and weight. Then, adolescents and mothers filled out self-report questionnaires. Based on mothers' reports, the Winkler social class index was computed as an indicator of families' SES ranging from 3-21 (Winkler \& Stolzenberg, 1999), with higher scores representing higher SES.

\section{Measures}

Adolescents

Perceived Parental Weight Teasing. A modified version of the Perception of Teasing Scale (POTS; Thompson et al., 1995; German version by AH - unpublished manuscript) was used to assess adolescents' perceived parental weight teasing by replacing "people" with "parents" as potential source of stigmatization in all 6 items $(1=$ never to $5=$ very often $)$. Higher sum scores indicated higher frequency of perceived parental weight teasing. Internal consistency was acceptable in this sample $(\alpha=.73)$. As the original two scales "frequency" 


\section{WEIGHT BIAS IN FAMILIES WITH BED}

describing the number of weight-related teasing experiences and "effect" describing the mental consequences of perceived weight related-teasing were highly correlated in the present study $(r=.96)$, we decided to focus on the frequency scale only.

Weight Bias Internalization. The Weight Bias Internalization Scale (WBIS; Durso \& Latner, 2008; German version: Hilbert, Baldofski, et al., 2014) measures the degree to which a respondent believes that negative stereotypes and self-statements about persons with overweight and obesity apply to herself or himself using 11 items $(1=$ strongly disagree, $7=$ strongly agree). Because of its negative correlation with the total mean score, Item 1 ("As an overweight person, I feel that I am just as competent as anyone.'”) was removed from the WBIS in accordance with Hilbert, Baldofski, et al. (2014). A total mean score was calculated, with higher scores indicating greater weight bias internalization $(\alpha=.87)$.

Eating Disorder Psychopathology. The Eating Disorder Examination-Questionnaire (EDE-Q; Fairburn \& Beglin, 1994; German version: Hilbert \& Tuschen-Caffier, 2016) was used to assess adolescents' specific eating disorder psychopathology within the past 28 days. The EDE-Q includes, besides six items on key behaviors, 22 items that are allocated to the subscales of restraint and eating concern, weight concern, and shape concern, were rated on a 6-point scale $(0=$ not at all to $6=$ markedly $)$. Based on these four subscales, a mean global score was calculated with higher values indicating greater psychopathology $(\alpha=.85)$.

Mothers

Maternal Stigmatizing Attitudes. The Attitudes Toward Obese Persons Scale (ATOP; Allison et al., 1991; German translation by $\mathrm{AH}$ - unpublished manuscript) assesses negative stereotypical attitudes toward obese persons. All 20 items were rated on a 6-point scale $(-3=I$ strongly disagree to $+3=$ I strongly agree). An overall ATOP sum score was computed (sum score +60 ), with higher scores indicating more positive attitudes toward obese persons. For the present sample, internal consistency was acceptable $(\alpha=.74)$. 


\section{WEIGHT BIAS IN FAMILIES WITH BED}

Maternal Stigmatizing Beliefs. The Beliefs About Obese Persons Scale (BAOP; Allison et al., 1991; German translation by AH - unpublished manuscript) is an 8-item measure $(-3=$ I strongly disagree to $+3=$ I strongly agree $)$ that measures beliefs about the controllability of obesity with 8 items. An overall BAOP sum score was computed (sum score $+24)$, with higher scores representing the belief that obesity is not within the control of the individual (Cronbach's $\alpha$ in the present study $=.67)$.

\section{Data Analytic Plan}

Statistical analyses were performed using SPSS 20.0 and PROCESS (Hayes, 2013). Hypothesized group differences in stigmatizing variables, meaning adolescents' perceived weight teasing (POTS), weight bias internalization (WBIS), maternal stigmatizing attitudes (ATOP) and beliefs (BAOP), were analyzed using one multivariate analyses of variances (MANOVA) to save power. In case of significance, univariate analyses with subsequent Bonferroni adjusted post-hoc tests were performed. Bivariate correlations were used to determine associations between adolescents' (POTS, WBIS) and maternal measures (ATOP, BAOP).

The proposed mediation model was calculated in PROCESS, an observed variable ordinary least squares regression path analysis, applying model 4 with 5000 bias-corrected bootstrap samples at a 95\% confidence interval to test direct and indirect effects. Perceived parental weight teasing (POTS) served as independent variable, weight bias internalization (WBIS) served as mediator, and global eating disorder psychopathology (EDE-Q) served as dependent variable.

Overall, significance level was set at a two-tailed $\alpha<0.05$ for all tests. Effect sizes were interpreted as small $\left(.01 \leq\right.$ partial $\left.\eta^{2}<.06 ; R^{2} \geq .01\right)$, medium $\left(.06 \leq\right.$ partial $\eta^{2}<.14 ; R^{2}$ $\geq .06$ ) or large (partial $\eta^{2} \geq .14 ; R^{2} \geq .14$ ). A post-hoc power analysis revealed that the statistical power to detect medium $\left(\mathrm{f}^{2}=.15, r=.30\right)$ and large $\left(\mathrm{f}^{2}=.35, r=.50\right)$ effects in the 


\section{WEIGHT BIAS IN FAMILIES WITH BED}

MANOVA and correlation analysis, respectively, was adequate to almost perfect $(1-\beta=$ $.97 / .84$ and $.99 / 1.00)$, given a significance level of $\alpha<0.05$.

\section{RESULTS}

Sample characteristics are reported in Table 1. Adolescents were predominantly female $(n=$ 71 girls, $78.9 \%), \chi^{2}(N=90, d f=2)=1.74, p>.05$, and primarily belonged to families with medium to high SES (22.9\% low SES; 39.8\% medium SES; $37.3 \%$ high SES), $\chi^{2}(N=90, d f=$ $3)=8.15, p>.05$. By design, weight status between groups differed between the BED and the NW group $(p<.001)$ as well as between the OW and the NW group $(p<.001)$. The BED group reported higher global eating disorder psychopathology than the OW $(p<.001)$ and NW $(p<.001)$ groups, and the OW revealed higher scores than the NW group $(p<.001)$. Mothers' weight status differed significantly between groups $\left(\chi^{2}(N=90, d f=6)=23.37, p<\right.$ $.001)$ with mothers of the NW group being more often classified as normal-weight (57\%) than those of the BED (35\%) and OW group (12\%), while the BED and OW did not differ significantly $(p=.195)$.

\section{Group Differences}

As hypothesized, a multivariate group effect was found for perceived parental weight teasing, weight bias internalization, maternal stigmatizing attitudes, and maternal stigmatizing beliefs, $F(8,148)=8.07, p<.001$. In univariate analyses, the BED group reported higher

perceived parental weight teasing (POTS) compared to both the OW $(p<.01)$ and NW groups $(p<.001)$, whereas the two latter did not differ significantly $(p>.05$; see Table 1$)$. Further, the BED group reported significantly higher weight bias internalization (WBIS) than the OW $(p<.001)$ and NW groups $(p<.001)$, and the OW revealed higher scores than the NW group $(p<.001)$. Maternal stigmatizing attitudes (ATOP) and maternal stigmatizing beliefs (BAOP) 


\section{WEIGHT BIAS IN FAMILIES WITH BED}

did not differ significantly between groups (all $p>.05$ ). Further, they were not significantly correlated with mothers' weight status $\left(r_{A T O P}=-.09, r_{B A O P}=.11\right.$, all $\left.p>.05\right)$.

\section{Bivariate Correlations between Adolescents' and Maternal Variables}

Contrary to hypothesis, perceived parental weight teasing and weight bias internalization were not significantly correlated with maternal stigmatizing attitudes $\left(r_{\text {POTS }}=\right.$ $.03, r_{\mathrm{WBIS}}=-.14$, all $\left.p>.05\right)$ and maternal stigmatizing beliefs $\left(r_{\mathrm{POTS}}=.14, r_{\mathrm{WBIS}}=-.12\right.$, all $p$ $>.05)$.

\section{Mediation Analysis of Weight Bias Internalization}

Adolescents' perceived parental weight teasing was a significant predictor for weight bias internalization, $F(1,82)=15.37, p<0.001$, adj $R^{2}=0.16 ; b=0.38, p<0.001,95 \% \mathrm{CI}$ $[0.42,0.62]$, indicating that adolescents' who experienced a higher frequency of perceived parental weight teasing reported higher weight bias internalization (see figure 1). In the direct effect model not accounting for weight bias internalization as a possible mediator, perceived parental weight teasing significantly predicted global eating disorder psychopathology, $F(1,82)=15.09, p<0.001, \operatorname{adj} R^{2}=0.16 ; b=0.26, p<0.001,95 \%$ CI $[0.13,0.40]$. The mediation model including weight bias internalization, showed that weight bias internalization fully mediated the relation between perceived parental weight teasing and adolescents' global eating disorder: perceived parental weight teasing was no longer a significant predictor of adolescents' global eating disorder after controlling for the mediator, $b$ $=0.06, p=0.20$. In the mediation model, the explained variance in adolescents' global eating disorder psychopathology increased substantially, $F(1,81)=71.17, p<0.001, \operatorname{adj} R^{2}=0.64$ (normal theory test for indirect effect: $Z=3.65, p<.001$ ). 


\section{WEIGHT BIAS IN FAMILIES WITH BED}

As maternal stigmatizing attitudes $(r=-.11, p>.05)$ and beliefs $(r=-.09, p>.05)$ were not correlated with adolescents' global eating disorder psychopathology, they were not included in the mediation analyses.

\section{DISCUSSION}

This study is the first to examine two sides of weight bias: adolescents' perceived parental weight teasing as well as maternal stigmatizing attitudes and beliefs and their impact on weight bias internalization and eating disorder psychopathology in adolescents with overweight and BED. First, as expected, we found higher perceived parental weight teasing and weight bias internalization in the BED group compared to overweight and normal-weight controls without BED. Second, adolescents' perceived parental weight teasing and maternal stigmatizing and beliefs attitudes were not correlated. Third, perceived parental weight teasing was identified as a significant predictor of adolescent global eating disorder psychopathology. However, its influence was substantially reduced when considering adolescents' level of weight bias internalization, which was found to fully mediate the association between perceived parental weight teasing and adolescents' global eating disorder psychopathology.

Most importantly, we provided evidence for the role of perceived weight teasing on eating disorder psychopathology in adolescents with overweight and BED. Our findings were in line with previous findings in adolescents with overweight (Neumark-Sztainer et al., 2002) and adults with BED (Hilbert, Pike, et al., 2014; Ashmore et al., 2008) showing that adolescents with overweight and BED were suffering from parental weight teasing to a greater extent when compared to adolescents with overweight or normal-weight and without BED. Further, our results supported evidence that parents may serve as an important source of weight teasing (Eisenberg et al., 2011) based on adolescents' perceptions, going in line with a previous finding in adolescent BED that the level of perceived maternal criticism predicted adolescents' global eating disorder psychopathology (Schmidt et al., 2015). 


\section{WEIGHT BIAS IN FAMILIES WITH BED}

This study initially examined weight bias internalization in adolescents with overweight and BED and explained that the group differences in weight bias internalization were not exclusively explained through adolescents' weight status, but were rather influenced by the presence of BED. Specifically, adolescents with overweight and BED were found to report greater weight bias internalization than adolescents with overweight only, which might be attributed to higher levels of eating disorder and general psychopathology (e.g., depression; Pearl et al., 2014) in those with BED. The associations of weight bias internalization and eating disorder psychopathology are well-known in adults with BED (Durso et al., 2012) and individuals with overweight and obesity (Pearl et al., 2014; Puhl et al., 2007). In this study, weight bias internalization fully mediated the association between perceived parental weight teasing and eating disorder psychopathology, which replicates a finding from a non-clinical student sample where the sources of weight teasing were not exclusively family members (O'Brien et al., 2016). Thus, adolescents with obesity and BED with a greater versus lower tendency to internalize weight bias are more affected by perceived parental weight teasing.

Contrary to expectations, we did not find a direct association between adolescents' perceived weight teasing, weight bias internalization, and maternal stigmatizing attitudes and beliefs. Limited by the study design, maternal measures did not directly assess stigmatizing attitudes about their children's weight status or their own weight-related parent-child interaction but rather focused on obesity in adolescents in general suggesting that both variables (adolescents' perceptions and maternal beliefs and attitudes) did not tap into the same construct, which may explain the lack of associations. Further, perceived parental teasing referred to both parents possibly reducing the associations with maternal attitudes and beliefs. However, the results mirror findings of a study in 9-year old girls documenting that measures of fat stereotypes were not correlated between girls and their parents (Davison \& Birch, 2004). Nevertheless, those girls were more likely to endorse fat stereotypes when 


\section{WEIGHT BIAS IN FAMILIES WITH BED}

interactions with parents focused on body shape and weight loss. Thus, it is possible that implicit stigmatizing attitudes are more explanatory of psychopathology than explicit stigmatizing attitudes. In adults with obesity and BED, experimental studies documented the predictive value of implicit weight bias for global eating disorder psychopathology in patients with obesity and BED using the Implicit Association Test (Greenwald et al., 1998; Brauhardt et al., 2014). Therefore, further research should focus on implicit measures to examine maternal stigmatizing attitudes and beliefs. Besides, other sources of teasing (e.g., by fathers, peers, or siblings) as well as other processes (e.g., adolescents' level of depression) might be relevant for the higher rates of perceived weight teasing and weight bias internalization in adolescents with overweight and BED.

\section{Strengths and Limitations}

A major strength of the study was the matching procedure that ruled out effects of sociodemographic variables including age, sex, weight status, and SES on the dependent variables in the sample. Additionally, a series of standardized measurements was used and a clinical expert interview confirmed BED diagnosis. Furthermore, this study was the first examining both perceived parental weight teasing assessed in adolescents as well as parental stigmatizing attitudes and beliefs assessed in the adolescents' mothers.

However, several limitations need to be accounted for when interpreting this study's findings. As this study firstly examined parental stigmatizing attitudes, we only focused on maternal perspectives due to reason of availability. Unfortunately, we cannot extrapolate on fathers' stigmatizing attitudes and their associations with adolescents' psychopathology. Further, the generalizability of findings is limited by the comparison of treatment-seeking and non-treatment-seeking groups and the oversampling of female participants. Although the measures used were validated in their English versions and in Spanish (POTS: LópezGuimerà et al., 2012), currently there is no published validation study for the POTS, ATOP, 


\section{WEIGHT BIAS IN FAMILIES WITH BED}

and BAOP in German available. Finally, the cross-sectional design precludes causal inferences regarding the impact of perceived parental weight teasing and weight bias internalization on eating disorder psychopathology. Regarding mediation analysis, we cannot certainly conclude that weight bias internalization mediates the effect of perceived teasing on eating disorder psychopathology or the other way around. Based on the literature, the first assumption is more likely to be the case, what definitely has to be evaluated using longitudinal, prospective data.

\section{Research and Clinical Implications}

So far, the availability of studies investigating perceived parental weight teasing and, especially, its association to parental stigmatizing attitudes in BED and overweight is limited, although family variables are meaningful for adolescents' well-being and might influence the development and maintenance of BED. Thus, more research is strongly warranted to further establish the role of perceived parental weight teasing in the development and maintenance of BED. As past research is limited to cross-sectional and retrospective designs in adolescent and adult samples (Neumark-Sztainer et al., 2002; Haines et al., 2006; Hilbert, Pike, et al., 2014), longitudinal studies will be needed to clarify causal relations. More generally, controlled samples measuring weight teasing in individuals with overweight with and without BED could give information about precise mechanisms of obesity and BED on teasing experiences. Finally, examinations should take into account the role of the father or other legal guardian as a potentially more powerful correlate of adolescents' perceptions, as well as other family or peer-group members.

Besides substantial future research implications, some clinical implications, sociallyand individually-oriented, can be derived from the present findings. As weight bias is a public health problem (Puhl \& Latner, 2007), it is clearly necessary to further establish interventions to reduce stigmatizing attitudes (for example, by providing causal information about obesity 


\section{WEIGHT BIAS IN FAMILIES WITH BED}

or cognitive dissonance interventions; O'Brien et al., 2011; Ciao \& Latner, 2010). In light of the current results on adolescents' perceptions, psychological interventions might not only target stigmatizing attitudes and perceptions (Hilbert \& Tuschen-Caffier, 2010), but could specifically address perceived weight teasing by family members. Therefore, psychological interventions for adolescents with BED should address important family factors by considering weight-related family interactions (e.g., during meals, buying food or clothes) and perceived family attitudes on weight and shape (Hilbert, 2013). In addition, the degree to which adolescents with BED internalize the weight bias and how they cope with weight teasing situations should be focused on. It is likely to be more difficult to reduce binge-eating behavior without addressing parental critical comments about weight and shape, if they act as a maintenance factor. 


\section{WEIGHT BIAS IN FAMILIES WITH BED}

\section{REFERENCES}

Allison, D. B., Basile, V. C., \& Yuker, H. E. (1991). The measurement of attitudes toward and beliefs about obese persons. International Journal of Eating Disorders, 10(5), 599-607.

American Psychiatric Association: DSM-5: Diagnostic and Statistical Manual of Mental Disorders. 5th edition. Arlington: American Psychiatric Association; 2013.

Ashmore, J. A., Friedman, K. E., Reichmann, S. K., \& Musante, G. J. (2008). Weightbased stigmatization, psychological distress, and binge eating behavior among obese treatment-seeking adults. Eating Behaviors, 9(2), 203-209.

Brauhardt, A., Rudolph, A., \& Hilbert, A. (2014). Implicit cognitive processes in bingeeating disorder and obesity. Journal of Behavior Therapy and Experimental Psychiatry, 45(2), 285-290.

Ciao, A. C., \& Latner, J. D. (2011). Reducing obesity stigma: the effectiveness of cognitive dissonance and social consensus interventions. Obesity, 19(9), 17681774.

Davison, K. K., \& Birch, L. L. (2004). Predictors of fat stereotypes among 9-year-old girls and their parents. Obesity Research, 12(1), 86-94.

Decaluwé, V., \& Braet, C. (2003). Prevalence of binge-eating disorder in obese children and adolescents seeking weight-loss treatment. International Journal of Obesity, 27(3), 404.

Decaluwé, V., Braet, C., \& Fairburn, C. G. (2003). Binge eating in obese children and adolescents. International Journal of Eating Disorders, 33(1), 78-84.

Durso, L. E., \& Latner, J. D. (2008). Understanding self-directed stigma: development of the weight bias internalization scale. Obesity, 16(S2). 


\section{WEIGHT BIAS IN FAMILIES WITH BED}

Durso, L. E., Latner, J. D., White, M. A., Masheb, R. M., Blomquist, K. K., Morgan, P. T., \& Grilo, C. M. (2012). Internalized weight bias in obese patients with binge eating disorder: associations with eating disturbances and psychological functioning. International Journal of Eating Disorders, 45(3), 423-427.

Eisenberg, M. E., Berge, J. M., Fulkerson, J. A., \& Neumark-Sztainer, D. (2011). Weight comments by family and significant others in young adulthood. Body Image, 8(1), 12-19.

Fairburn, C. G. \& Beglin, S. J. (1994). The assessment of eating disorders: Interview or self-report questionnaire? International Journal of Eating Disorders, 16(4), 363370.

Fairburn, C. G., Cooper, Z. \& O’Connor, M. E. (2008). Eating disorder examination (edition 16.0D). In: Fairburn, C.G. (Ed.), Cognitive Behavior Therapy and Eating Disorders. Guilford Press, New York, 265-309.

Fairburn, C. G., \& Harrison, P. J. (2003). Eating disorders. The Lancet, 361(9355), 407416.

Greenwald, A. G., McGhee, D. E., \& Schwartz, J. L. (1998). Measuring individual differences in implicit cognition: the implicit association test. Journal of Personality and Social Psychology, 74(6), 1464-1480.

Haines, J., Neumark-Sztainer, D., Eisenberg, M. E., \& Hannan, P. J. (2006). Weight teasing and disordered eating behaviors in adolescents: longitudinal findings from Project EAT (Eating Among Teens). Pediatrics, 117(2), e209-e215.

Hayes, A. F. (2013). Introduction to mediation, moderation, and conditional process analysis: A regression-based approach. New York, NY: The Guilford Press.

Hilbert, A. (2013). Cognitive-behavioral therapy for binge eating disorder in adolescents: study protocol for a randomized controlled trial. Trials, 14(312), 1-11. 


\section{WEIGHT BIAS IN FAMILIES WITH BED}

Hilbert, A., Baldofski, S., Zenger, M., Löwe, B., Kersting, A., \& Braehler, E. (2014). Weight bias internalization scale: psychometric properties and population norms. PloSone, 9(1), e86303.

Hilbert, A., Braehler, E., Haeuser, W., \& Zenger, M. (2014). Weight bias internalization, core self - evaluation, and health in overweight and obese persons. Obesity, 22(1), $79-85$.

Hilbert, A., Pike, K. M., Goldschmidt, A. B., Wilfley, D. E., Fairburn, C. G., Dohm, F. A., \& Walsh, B. T. (2014). Risk factors across the eating disorders. Psychiatry Research, 220(1), 500-506.

Hilbert, A., \& Tuschen-Caffier, B. (2006). Eating Disorder Examination. Deutschsprachige Übersetzung. Münster: Verlag für Psychotherapie.

Hilbert, A., \& Tuschen-Caffier, B. (2010). Essanfälle und Adipostas: Ein Manual zur kognitiv-behavioralen Therapie der Binge-Eating-Störung [Binge Eating and Obesity: Cognitive-Behavioral Therapy Manual for Binge Eating Disorder]. Goettingen: Hogrefe.

Hilbert, A., \& Tuschen-Caffier, B. (2016). Eating Disorder Examination-Questionnaire. Deutschsprachige Übersetzung (2. Auflage). Tübingen: Deutsche Gesellschaft für Verhaltenstherapie (DGVT).

Hilbert, A., Tuschen-Caffier, B., Karwautz, A., Niederhofer, H., \& Munsch, S. (2007). Eating Disorder Examination-Questionnaire. Diagnostica, 53(3), 144-154.

Hübner, C., Baldofski, S., Zenger, M., Tigges, W., Herbig, B., Jurowich, C., ... \& Hilbert, A. (2015). Influences of general self-efficacy and weight bias internalization on physical activity in bariatric surgery candidates. Surgery for Obesity and Related Diseases, 11(6), 1371-1376.

Kraus, S. J. (1995). Attitudes and the prediction of behavior: a meta-analysis of the empirical literature. Personality and Social Psychology Bulletin, 21(1), 58-75. 


\section{WEIGHT BIAS IN FAMILIES WITH BED}

Libbey, H. P., Story, M. T., Neumark-Sztainer, D. R., \& Boutelle, K. N. (2008). Teasing, disordered eating behaviors, and psychological morbidities among overweight adolescents. Obesity, 16(S2), S24-S29.

López-Guimerà, G., Fauquet, J., Sánchez-Carracedo, D., Barrada, J. R., Saldaña, C., \& Masnou-Roig, A. (2012). Psychometric properties of the perception of teasing scale in a Spanish adolescent sample: POTS-S. Eating and Weight Disorders, 17(3), e210-e218.

Neumark-Sztainer, D., Falkner, N., Story, M., Perry, C., \& Hannan, P. J. (2002). Weightteasing among adolescents: correlations with weight status and disordered eating behaviors. International Journal of Obesity and Related Metabolic Disorders, 26(1), 123-131.

O'Brien, K. S., Latner, J. D., Puhl, R. M., Vartanian, L. R., Giles, C., Griva, K., \& Carter, A. (2016). The relationship between weight stigma and eating behavior is explained by weight bias internalization and psychological distress. Appetite, 102, 70-76.

O'Brien, K. S., Puhl, R. M., Latner, J. D., Mir, A. S., \& Hunter, J. A. (2010). Reducing anti-fat prejudice in preservice health students: a randomized trial. Obesity, 18(11), 2138-2144.

Pearl, R. L., White, M. A., \& Grilo, C. M. (2014). Weight bias internalization, depression, and self-reported health among overweight binge eating disorder patients. Obesity, 22(5), E142-E148.

Puhl, R. M., \& Brownell, K. D. (2003). Psychosocial origins of obesity stigma: toward changing a powerful and pervasive bias. Obesity Reviews, 4(4), 213-227.

Puhl, R. M., \& Brownell, K. D. (2006). Confronting and coping with weight stigma: an investigation of overweight and obese adults. Obesity, 14(10), 1802-1815. 


\section{WEIGHT BIAS IN FAMILIES WITH BED}

Puhl, R. M., \& Heuer, C. A. (2009). The stigma of obesity: a review and update. Obesity, 17(5), 941-964.

Puhl, R. M., \& King, K. M. (2013). Weight discrimination and bullying. Clinical Endocrinology and Metabolism, 27(2), 117-127.

Puhl, R. M., \& Latner, J. D. (2007). Stigma, obesity, and the health of the nation's children. Psychological Bulletin, 133(4), 557-580.

Puhl, R. M., Moss-Racusin, C. A., \& Schwartz, M. B. (2007). Internalization of weight bias: implications for binge eating and emotional well-being. Obesity, 15(1), 1923.

Puhl, R., \& Suh, Y. (2015). Stigma and eating and weight disorders. Current Psychiatry Reports, 17(3), 1-10.

Repetti, R. L., Taylor, S. E., \& Seeman, T. E. (2002). Risky families: family social environments and the mental and physical health of offspring. Psychological Bulletin, 128(2), 330-366.

Schmidt, R., Tetzlaff, A., \& Hilbert, A. (2015). Perceived expressed emotion in adolescents with binge-eating disorder. Journal of Abnormal Child Psychology, 43(7), 1369-1377.

Sienko, R. M., Saules, K. K., \& Carr, M. M. (2016). Internalized weight bias mediates the relationship between depressive symptoms and disordered eating behavior among women who think they are overweight. Eating Behaviors, 22, 141-144.

Swanson, S. A., Crow, S. J., Le Grange, D, Swendsen J., \& Merikangas, K. R. (2011). Prevalence and correlates of eating disorders in adolescents. Results from the national comorbidity survey replication adolescent supplement. Archives of General Psychiatry, 68(7), 714-723. 


\section{WEIGHT BIAS IN FAMILIES WITH BED}

Thompson, J. K., Cattarin, J., Fowler, B., \& Fisher, E. (1995). The perception of teasing scale (POTS): a revision and extension of the physical appearance related teasing scale (PARTS). Journal of Personality Assessment, 65(1), 146-157.

Ventura, A. K., \& Birch, L. L. (2008). Does parenting affect children's eating and weight status? International Journal of Behavioral Nutrition and Physical Activity, 5(15), $1-12$.

Wabitsch, M., Kunze, D. (2015). Konsensbasierte (S2) Leitlinie zur Diagnostik, Therapie und Prävention von Übergewicht und Adipositas im Kindes- und Jugendalter [Evidence-based therapy guideline of the German Working Group on Obesity in Childhood and Adolescence]. Retrieved September 27, 2017 from www.a-g-a.de.

Winkler, J., \& Stolzenberg, H. (1999). Der Schichtindex 1998 [Social class index in the Federal Health Survey]. Gesundheitswesen, 61, 177-182.

Zuba, A., \& Warschburger, P. (2017). The role of weight teasing and weight bias internalization in psychological functioning: a prospective study among schoolaged children. European Child and Adolescent Psychiatry, 1-11. 
Table 1

Sample characteristics and group differences in adolescents' perceived parental weight teasing, weight bias internalization, and maternal stigmatizing attitudes

\begin{tabular}{|c|c|c|c|c|}
\hline $\operatorname{BED}(n=40)$ & OW $(n=25)$ & $\mathrm{NW}(n=25)$ & ANOVA & $\eta^{2}$ \\
\hline$M(S D)$ & $M(S D)$ & $M(S D)$ & & \\
\hline
\end{tabular}

\begin{tabular}{|c|c|c|c|c|c|}
\hline \multicolumn{6}{|l|}{ Sociodemographic and Clinical Variables } \\
\hline Age (years) & $14.58(2.39)$ & $14.53(2.55)$ & $15.84(2.67)$ & $F(2,88)=2.35$ & 0.05 \\
\hline BMI-SDS & $2.25(0.64)^{\mathrm{a}}$ & $2.38(0.59)^{\mathrm{a}}$ & $0.45(0.56)^{b}$ & $F(2,89)=69.59 * * *$ & 0.65 \\
\hline Eating disorder psychopathology (EDE-Q) & $2.59(0.95)^{\mathrm{a}}$ & $1.75(1.24)^{\mathrm{b}}$ & $0.84(0.78)^{\mathrm{c}}$ & $F(2,88)=23.74 * * *$ & 0.36 \\
\hline Maternal BMI (kg/m²) & $28.17(7.03)^{\mathrm{a}}$ & $32.10(6.65)^{\mathrm{b}}$ & $23.86(3.54)^{\mathrm{c}}$ & $F(2,87)=11.07 * * *$ & 0.21 \\
\hline \multicolumn{6}{|l|}{ Variables of Weight Bias } \\
\hline Perceived parental weight teasing (POTS) & $7.68(2.38)^{\mathrm{a}}$ & $6.40(0.91)^{b}$ & $6.08(0.40)^{\mathrm{b}}$ & $F(2,89)=8.37 * * *$ & 0.16 \\
\hline Weight bias internalization (WBIS) & $5.12(1.26)^{\mathrm{a}}$ & $3.42(1.44)^{\mathrm{b}}$ & $2.13(1.09)^{\mathrm{c}}$ & $F(2,84)=39.17 * * *$ & 0.49 \\
\hline Maternal stigmatizing attitudes (ATOP) & $68.38(15.72)$ & $73.00(14.42)$ & $72.44(16.51)$ & $F(2,87)=0.84$ & 0.02 \\
\hline Maternal stigmatizing beliefs (BAOP) & $16.95(7.26)$ & $19.08(7.56)$ & $15.48(6.72)$ & $F(2,88)=1.59$ & 0.04 \\
\hline
\end{tabular}




\section{WEIGHT BIAS IN FAMILIES WITH BED}

Note . $\mathrm{BED}=$ adolescents with overweight and binge-eating disorder; $\mathrm{OW}=$ adolescents with overweight and without binge-eating disorder; $\mathrm{NW}=$ normal-weight control group; BMI-SDS = standard deviation score of the body mass index; BMI = body mass index; EDE-Q = Eating Disorder Examination-Questionnaire; POTS = Perception of Teasing Scale; WBIS = Weight Bias Internalization Scale; ATOP = Attitudes Toward Obese Persons Scale; BAOP = Beliefs About Obese Persons Scale.

${ }^{a, b, c}$ Different superscripts indicate significant group differences.

$* * * p<.001$ 
Figure 1. Mediation Analysis of Weight Bias Internalization

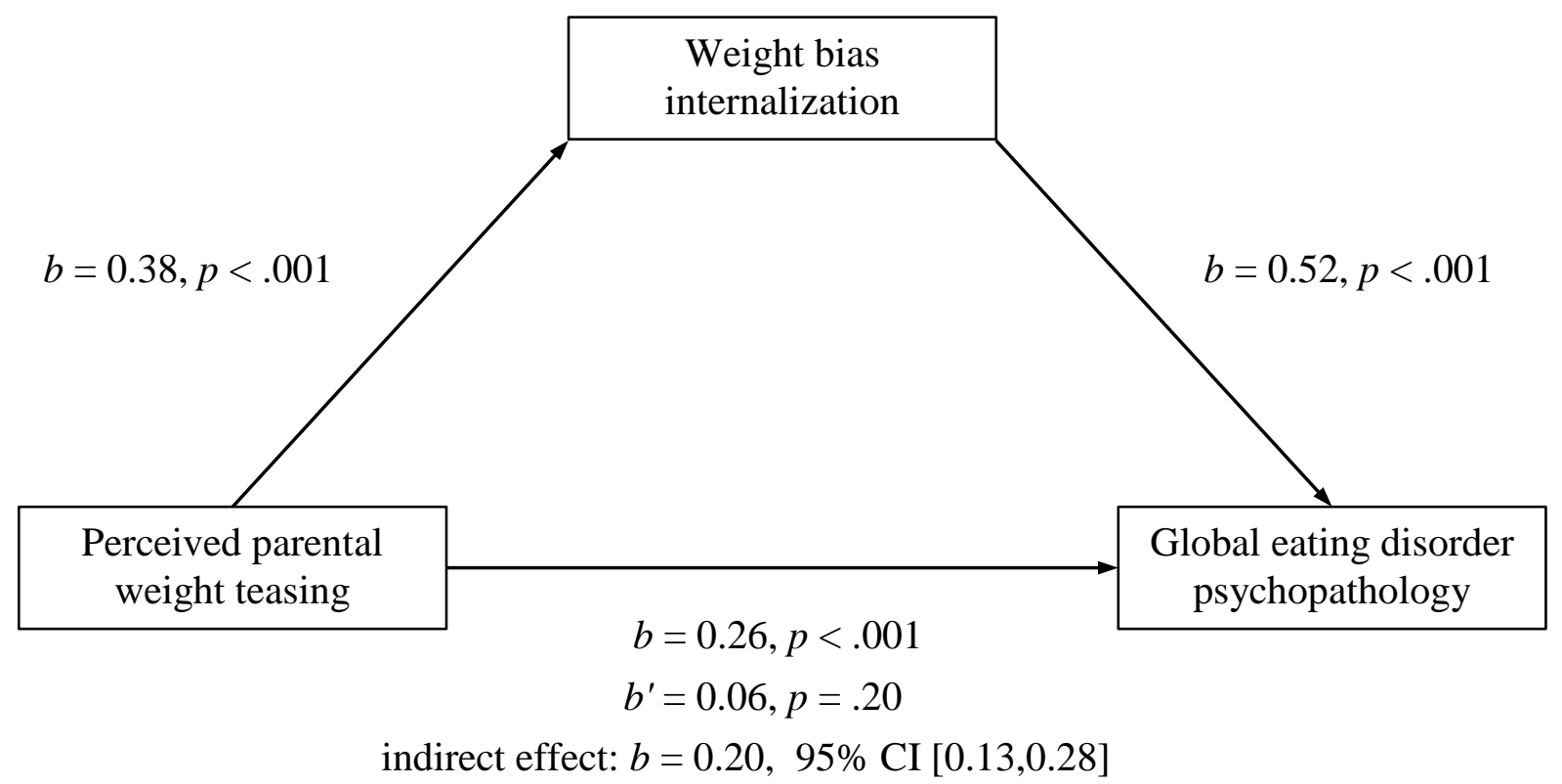

Figure 1. Mediation model with weight bias internalization as a mediator of the relation between adolescents' perceived parental weight teasing and global eating disorder psychopathology. $b^{\prime}$ indicates the direct effect of perceived parental weight teasing on global eating disorder psychopathology when controlled for the indirect effect of the mediation. 